CRYSTALLOGRAPHIC COMMUNICATIONS

ISSN 2056-9890

Received 23 December 2015

Accepted 4 January 2016

Edited by $\mathrm{H}$. Stoeckli-Evans, University of Neuchâtel, Switzerland

\section{Crystal structure of 1-benzyl-4-formyl-1H-pyrrole- 3-carboxamide}

\author{
Qi-Di Zhong, Sheng-Quan Hu and Hong Yan*
}

College of Life Science and Bio-Engineering, Beijing University of Technology, 100124 Chaoyang District, Beijing, People's Republic of China. *Correspondence e-mail: hongyan@bjut.edu.cn

In the title compound, $\mathrm{C}_{13} \mathrm{H}_{12} \mathrm{~N}_{2} \mathrm{O}_{2}$ (I), the mean planes of the pyrrole and benzyl rings are approximately perpendicular, forming a dihedral angle of 87.07 (4) ${ }^{\circ}$. There is an intramolecular $\mathrm{N}-\mathrm{H} \cdots \mathrm{O}$ hydrogen bond forming an $\mathrm{S}(7)$ ring motif. In the crystal, molecules are linked via a pair of $\mathrm{N}-\mathrm{H} \cdots \mathrm{O}$ hydrogen bonds forming inversion dimers. $\mathrm{C}-\mathrm{H} \cdots \mathrm{O}$ hydrogen bonds link the dimers into chains along direction [101] . The chains are further linked by weak $\mathrm{C}-\mathrm{H} \cdots \pi$ interactions forming layers parallel to the ac plane.

\section{Chemical context}

Pyrrole and its derivatives are classes of heterocyclic compounds and that have attracted much attention because of their potential pharmacological and biological properties (Davis et al., 2008; Meshram et al., 2010; Moriguchi et al., 2015). As a part of our work on the synthesis of new pyrrole derivatives with good biological activities, the title compound, (I), was synthesized and its crystal structure is reported on herein.

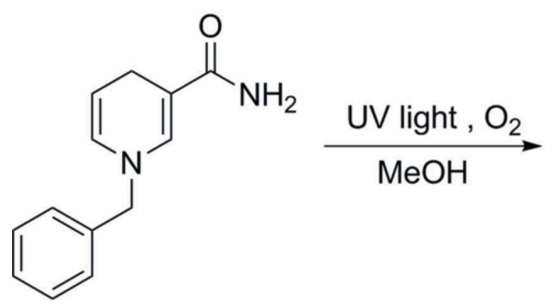<smiles>NC(=O)c1cn(Cc2ccccc2)cc1C=O</smiles>

(I)

\section{Structural commentary}

The molecular structure of the title compound (I), is shown in Fig. 1. In the amide group, the $\mathrm{C}-\mathrm{N}$ bond is relatively short

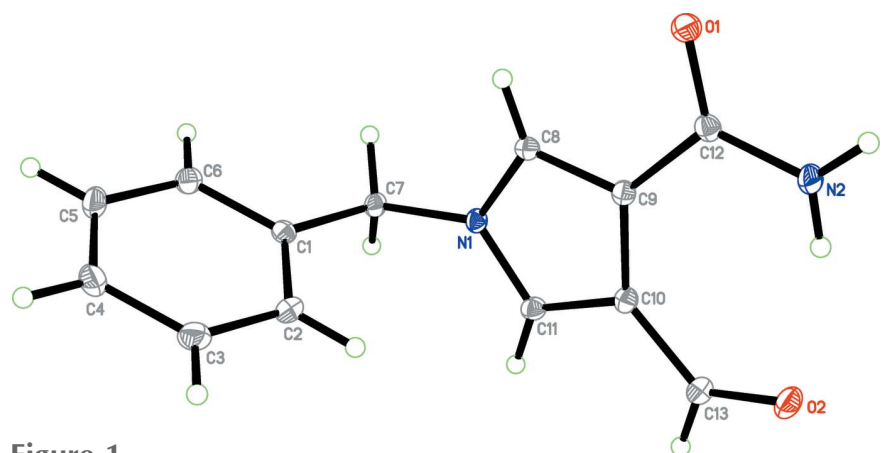

Figure 1

A view of the molecular structure of the title compound (I), with atom labelling. Displacement ellipsoids are drawn at the $30 \%$ probability level. 
Table 1

Hydrogen-bond geometry $\left(\AA,^{\circ}\right)$.

$C g 1$ is the centroid of the benzyl ring $\mathrm{C} 1-\mathrm{C} 6$.

\begin{tabular}{lllll}
\hline$D-\mathrm{H} \cdots A$ & $D-\mathrm{H}$ & $\mathrm{H} \cdots A$ & $D \cdots A$ & $D-\mathrm{H} \cdots A$ \\
\hline $\mathrm{N} 2-\mathrm{H} 2 B \cdots \mathrm{O} 2$ & 0.86 & 1.99 & $2.8184(14)$ & 160 \\
$\mathrm{~N} 2-\mathrm{H} 2 A \cdots \mathrm{O} 1^{\mathrm{i}}$ & 0.86 & 2.22 & $3.0063(14)$ & 151 \\
$\mathrm{C} 8-\mathrm{H} 8 \cdots \mathrm{O} 1^{\mathrm{ii}}$ & 0.93 & 2.69 & $3.4252(15)$ & 136 \\
$\mathrm{C} 7-\mathrm{H} 7 B \cdots \mathrm{O} 1^{\mathrm{ii}}$ & 0.97 & 2.48 & $3.3123(15)$ & 144 \\
$\mathrm{C} 7-\mathrm{H} 7 A \cdots \mathrm{O}{ }^{\mathrm{ii}}$ & 0.97 & 2.66 & $3.3268(15)$ & 126 \\
$\mathrm{C} 11-\mathrm{H} 11 \cdots C g 1^{\text {iv }}$ & 0.93 & 2.58 & $3.4962(14)$ & 167 \\
\hline
\end{tabular}

Symmetry codes: (i) $\quad-x+1,-y+2,-z-1$; $\quad$ (ii) $\quad-x+2,-y+2,-z$; (iii) $x+1, y, z+1 ;$ (iv) $x-1, y, z$.

[C12-N2 = $1.3374(16) \AA]$, suggesting some degree of electronic delocalization in the molecule. The dihedral angle between the pyrrole and phenyl rings is $87.07(4)^{\circ}$, indicating that they are nearly perpendicular to each other. An intramolecular hydrogen bond, $\mathrm{N} 2-\mathrm{H} 2 B \cdots \mathrm{O} 2$ (Table 1), encloses an $S(7)$ ring motif.

\section{Supramolecular features}

In the crystal of (I), $\mathrm{N} 2-\mathrm{H} 2 A \cdots \mathrm{O} 1^{\mathrm{i}}$ hydrogen bonds [symmetry code: (i) $-x+1,-y+2,-z-1$ ], link pairs of molecules, forming inversion dimers with an $R_{2}^{2}(8)$ ring motif (Table 1 and Fig. 2). The dimers are further linked by $\mathrm{C} 7-$ $\mathrm{H} 7 B \cdots \mathrm{O} 1^{\mathrm{ii}}, \mathrm{C} 8-\mathrm{H} 8 \cdots \mathrm{O} 1^{\mathrm{ii}}$ and $\mathrm{C} 7-\mathrm{H} 7 A \cdots \mathrm{O} 2^{\mathrm{iii}}$ hydrogen

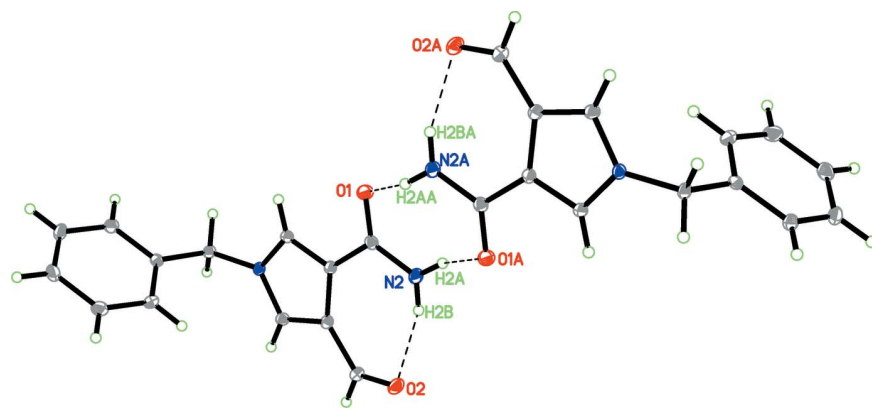

Figure 2

A view of the inversion dimer formed by pairs of $\mathrm{N}-\mathrm{H} \cdots \mathrm{O}$ hydrogen bonds. Both the intramolecular and intermolecular hydrogen bonds are shown as dashed lines (see Table 1).

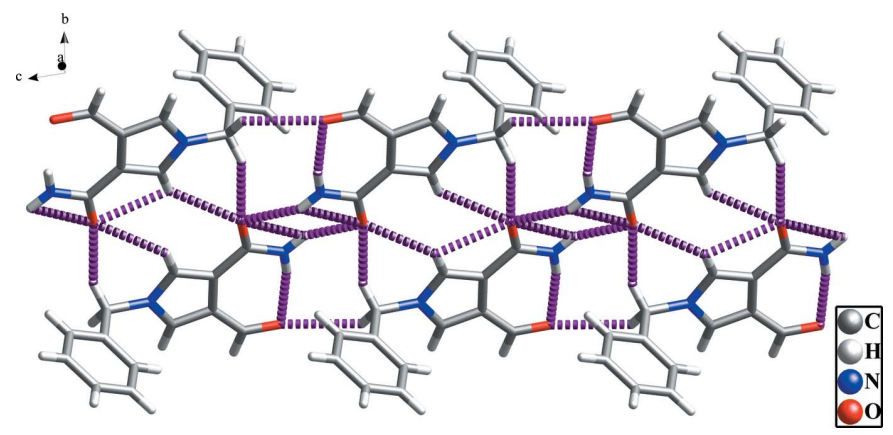

Figure 3

A view of the one-dimensional chain structure. The dashed lines indicate the $\mathrm{N}-\mathrm{H} \cdots \mathrm{O}$ and $\mathrm{C}-\mathrm{H} \cdots \mathrm{O}$ hydrogen bonds (see Table 1 ).

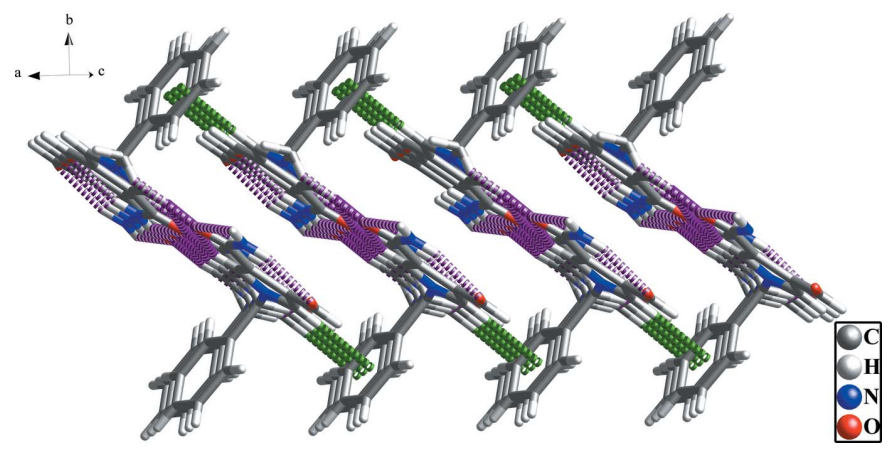

Figure 4

The view of the two-dimensional network structure. The $\mathrm{C}-\mathrm{H} \cdots \pi$ interactions and the hydrogen bonds are shown with green and purple dashed lines, respectively (see Table 1).

bonds [symmetry codes: (ii) $-x+2,-y+2,-z$; (iii) $x+1, y$,

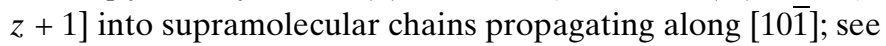
Table 1 and Fig. 3). Adjacent chains are linked by weak C11$\mathrm{H} 11 \cdots C g 1^{\text {iv }}$ contacts $[C g 1$ is the centroid of the $\mathrm{C} 1-\mathrm{C} 6$ benzyl ring; symmetry code: (iv) $-1+x, y, z$ ], forming layers parallel to the $a c$ plane (Table 1 and Fig. 4).

\section{Database survey}

A search of the Cambridge Structural Database (Version 5.36 with three updates; Groom \& Allen, 2014) for 1-benzyl-4formyl-1H-pyrrole-3-carboxamide gave no hits. However, structures of substituted derivatives of 1-benzyl-1H-pyrrole

Table 2

Experimental details

\begin{tabular}{ll}
\hline Crystal data & \\
Chemical formula & $\mathrm{C}_{13} \mathrm{H}_{12} \mathrm{~N}_{2} \mathrm{O}_{2}$ \\
$M_{\mathrm{r}}$ & 228.25 \\
Crystal system, space group & Monoclinic, $P 2_{1} / c$ \\
Temperature $(\mathrm{K})$ & 293 \\
$a, b, c(\AA)$ & $5.5296(6), 23.083(3), 9.3088(9)$ \\
$\beta\left({ }^{\circ}\right.$ & $112.940(5)$ \\
$V\left(\AA^{3}\right)$ & $1094.2(2)$ \\
$Z$ & 4 \\
Radiation type & Mo $K \alpha$ \\
$\mu\left(\mathrm{mm}^{-1}\right)$ & 0.10 \\
Crystal size (mm) & $0.25 \times 0.20 \times 0.18$ \\
& \\
Data collection & Bruker SMART CCD area \\
Diffractometer & detector \\
& Multi-scan $(S A D A B S ;$ Bruker, \\
Absorption correction & $2005)$ \\
& $0.977,0.983$ \\
$T_{\text {min }}, T_{\text {max }}$ & $9372,1938,1823$ \\
No. of measured, independent and & \\
$\quad$ observed $[I>2 \sigma(I)]$ reflections & 0.021 \\
$R_{\text {int }}$ & 0.596 \\
(sin $\theta / \lambda)_{\text {max }}\left(\AA^{-1}\right)$ & \\
Refinement & \\
$R\left[F^{2}>2 \sigma\left(F^{2}\right)\right], w R\left(F^{2}\right), S$ & $0.034,0.123,1.00$ \\
No. of reflections & 1938 \\
No. of parameters & 154 \\
$\mathrm{H}$-atom treatment & $\mathrm{H}$-atom parameters constrained \\
$\Delta \rho_{\text {max }}, \Delta \rho_{\text {min }}\left(\mathrm{e} \AA^{-3}\right)$ & $0.19,-0.26$ \\
\hline &
\end{tabular}

Computer programs: APEX2 and SAINT (Bruker, 2005) and SHELXTL (Sheldrick, 2008). 
were found, see for example Bonnett et al. (1985); Choi et al. (1998); Sha et al. (1990); Wang et al. (2011). In these structures, the pyrrole and benzyl rings are also nearly perpendicular to one another.

\section{Synthesis and crystallization}

1-Benzyl-1 $H$-pyrrole-3-carboxamide $(1 \mathrm{mmol}, 214.3 \mathrm{mg})$ was dissolved in methanol $(20 \mathrm{ml})$ and irradiated with UV light at room temperature under oxygen (see Scheme). The reaction progress was monitored by thin layer chromatography (TLC). After completion, the solvent was removed under reduced pressure, and the residue was purified by chromatography on silica gel, using a mixed solvent of petroleum ether and ethyl acetate $(10: 1$ ratio, $v / v)$, to give the pure product. Colourless single crystals, suitable for X-ray diffraction analysis, were obtained by slow evaporation of a methanol solution of the title compound at room temperature.

\section{Refinement}

Crystal data, data collection and structure refinement details are summarized in Table 2. All $\mathrm{H}$ atoms were placed in idealized positions $(\mathrm{C}-\mathrm{H}=0.93-0.97 \AA, \mathrm{N}-\mathrm{H}=0.86 \AA)$ and refined as riding atoms, with $U_{\text {iso }}(\mathrm{H})=1.2 U_{\text {eq }}(\mathrm{N}, \mathrm{C})$.

\section{Acknowledgements}

This work was supported financially by the Key Projects in the Beijing Municipal Natural Science Foundation (No. KZ201510005007).

\section{References}

Bonnett, R., Hursthouse, M. B., North, S. A. \& Trotter, J. (1985). J. Chem. Soc. Perkin Trans. 2, pp. 293-296.

Bruker (2005). APEX2, SAINT and SADABS. Bruker AXS Inc., Madison, Wisconsin, USA.

Choi, D. S., Huang, S., Huang, M., Barnard, T. S., Adams, R. D., Seminario, J. M. \& Tour, J. M. (1998). J. Org. Chem. 63, 26462655.

Davis, F. A., Bowen, K., Xu, H. \& Velvadapu, V. (2008). Tetrahedron, 64, 4174-4182.

Meshram, H. M., Prasad, B. R. V. \& Kumar, D. A. (2010). Tetrahedron Lett. 51, 3477-3480.

Moriguchi, T., Jalli, V., Krishnamurthy, S., Tsuge, A. \& Yoza, K. (2015). Acta Cryst. E71, o1049-o1050.

Sha, C. K., Liu, J. M., Chiang, R. K. \& Wang, S. L. (1990). Heterocycles, 31, 603-609.

Sheldrick, G. M. (2008). Acta Cryst. A64, 112-122.

Wang, Z., Li, K., Zhao, D., Lan, J. \& You, J. (2011). Angew. Chem. Int. Ed. 50, 5365-5369. 


\section{supporting information}

Acta Cryst. (2016). E72, 133-135 [doi:10.1107/S2056989016000128]

\section{Crystal structure of 1-benzyl-4-formyl-1H-pyrrole-3-carboxamide}

\section{Qi-Di Zhong, Sheng-Quan Hu and Hong Yan}

\section{Computing details}

Data collection: APEX2 (Bruker, 2005); cell refinement: SAINT (Bruker, 2005); data reduction: SAINT (Bruker, 2005); program(s) used to solve structure: SHELXTL (Sheldrick, 2008); program(s) used to refine structure: SHELXTL (Sheldrick, 2008); molecular graphics: SHELXTL (Sheldrick, 2008) (Sheldrick, 2008); software used to prepare material for publication: SHELXTL.

1-Benzyl-4-formyl-1H-pyrrole-3-carboxamide

Crystal data

$\mathrm{C}_{13} \mathrm{H}_{12} \mathrm{~N}_{2} \mathrm{O}_{2}$

$M_{r}=228.25$

Monoclinic, $P 2{ }_{1} / c$

Hall symbol: -P 2ybc

$a=5.5296(6) \AA$

$b=23.083(3) \AA$

$c=9.3088(9) \AA$

$\beta=112.940(5)^{\circ}$

$V=1094.2(2) \AA^{3}$

$Z=4$

\section{Data collection}

Bruker SMART CCD area-detector diffractometer

Radiation source: fine-focus sealed tube Graphite monochromator

phi and $\omega$ scans

Absorption correction: multi-scan

(SADABS; Bruker, 2005)

$T_{\min }=0.977, T_{\max }=0.983$

\section{Refinement}

Refinement on $F^{2}$

Least-squares matrix: full

$R\left[F^{2}>2 \sigma\left(F^{2}\right)\right]=0.034$

$w R\left(F^{2}\right)=0.123$

$S=1.00$

1938 reflections

154 parameters

0 restraints

Primary atom site location: structure-invariant direct methods
$F(000)=480$

$D_{\mathrm{x}}=1.386 \mathrm{Mg} \mathrm{m}^{-3}$

Mo $K \alpha$ radiation, $\lambda=0.71073 \AA$

Cell parameters from 3196 reflections

$\theta=3.5-27.5^{\circ}$

$\mu=0.10 \mathrm{~mm}^{-1}$

$T=293 \mathrm{~K}$

Block, colorless

$0.25 \times 0.20 \times 0.18 \mathrm{~mm}$

9372 measured reflections

1938 independent reflections

1823 reflections with $I>2 \sigma(I)$

$R_{\text {int }}=0.021$

$\theta_{\max }=25.1^{\circ}, \theta_{\min }=3.5^{\circ}$

$h=-6 \rightarrow 6$

$k=-27 \rightarrow 27$

$l=-11 \rightarrow 11$

Secondary atom site location: difference Fourier map

Hydrogen site location: inferred from neighbouring sites

$\mathrm{H}$-atom parameters constrained

$w=1 /\left[\sigma^{2}\left(F_{\mathrm{o}}^{2}\right)+(0.1 P)^{2}+0.2118 P\right]$

where $P=\left(F_{\mathrm{o}}^{2}+2 F_{\mathrm{c}}{ }^{2}\right) / 3$

$(\Delta / \sigma)_{\max }=0.001$

$\Delta \rho_{\max }=0.19 \mathrm{e} \AA^{-3}$

$\Delta \rho_{\min }=-0.26$ e $\AA^{-3}$ 


\section{Special details}

Geometry. All e.s.d.'s (except the e.s.d. in the dihedral angle between two 1.s. planes) are estimated using the full covariance matrix. The cell e.s.d.'s are taken into account individually in the estimation of e.s.d.'s in distances, angles and torsion angles; correlations between e.s.d.'s in cell parameters are only used when they are defined by crystal symmetry. An approximate (isotropic) treatment of cell e.s.d.'s is used for estimating e.s.d.'s involving 1.s. planes.

Refinement. Refinement of $F^{2}$ against ALL reflections. The weighted $R$-factor $w R$ and goodness of fit $S$ are based on $F^{2}$, conventional $R$-factors $R$ are based on $F$, with $F$ set to zero for negative $F^{2}$. The threshold expression of $F^{2}>\sigma\left(F^{2}\right)$ is used only for calculating $R$-factors (gt) etc. and is not relevant to the choice of reflections for refinement. $R$-factors based on $F^{2}$ are statistically about twice as large as those based on $F$, and $R$ - factors based on ALL data will be even larger.

Fractional atomic coordinates and isotropic or equivalent isotropic displacement parameters $\left(\AA^{2}\right)$

\begin{tabular}{|c|c|c|c|c|}
\hline & $x$ & $y$ & $z$ & $U_{\text {iso }} * / U_{\text {eq }}$ \\
\hline $\mathrm{C} 1$ & $1.0335(2)$ & $0.84645(5)$ & $0.26085(13)$ & 0.0165 \\
\hline $\mathrm{C} 2$ & $0.9577(2)$ & $0.80281(5)$ & $0.14829(15)$ & 0.0217 \\
\hline $\mathrm{H} 2$ & 0.7904 & 0.8035 & 0.0696 & $0.026^{*}$ \\
\hline $\mathrm{C} 3$ & $1.1293(3)$ & $0.75831(6)$ & $0.15255(17)$ & 0.0267 \\
\hline $\mathrm{H} 3$ & 1.0774 & 0.7298 & 0.0760 & $0.032 *$ \\
\hline $\mathrm{C} 4$ & $1.3792(3)$ & $0.75629(6)$ & $0.27120(17)$ & 0.0271 \\
\hline $\mathrm{H} 4$ & 1.4942 & 0.7264 & 0.2746 & $0.033^{*}$ \\
\hline $\mathrm{C} 5$ & $1.4545(2)$ & $0.79918(6)$ & $0.38396(15)$ & 0.0252 \\
\hline H5 & 1.6204 & 0.7979 & 0.4640 & $0.030^{*}$ \\
\hline C6 & $1.2845(2)$ & $0.84412(5)$ & $0.37859(14)$ & 0.0206 \\
\hline H6 & 1.3385 & 0.8730 & 0.4543 & $0.025^{*}$ \\
\hline $\mathrm{C} 7$ & $0.8542(2)$ & $0.89645(5)$ & $0.25832(13)$ & $0.0167(3)$ \\
\hline H7A & 0.7595 & 0.8869 & 0.3236 & $0.020^{*}$ \\
\hline H7B & 0.9601 & 0.9305 & 0.3022 & $0.020^{*}$ \\
\hline $\mathrm{C} 8$ & $0.7226(2)$ & $0.94298(5)$ & $-0.00412(13)$ & 0.0158 \\
\hline $\mathrm{H} 8$ & 0.8792 & 0.9627 & 0.0164 & $0.019^{*}$ \\
\hline C9 & $0.5118(2)$ & $0.94220(5)$ & $-0.14451(13)$ & $0.0152(3)$ \\
\hline $\mathrm{C} 10$ & $0.3150(2)$ & $0.90594(5)$ & $-0.12295(13)$ & $0.0162(3)$ \\
\hline $\mathrm{C} 11$ & $0.4214(2)$ & $0.88767(5)$ & $0.03064(13)$ & $0.0171(3)$ \\
\hline H11 & 0.3383 & 0.8638 & 0.0779 & $0.021 *$ \\
\hline $\mathrm{C} 12$ & $0.5128(2)$ & $0.97561(5)$ & $-0.28041(13)$ & 0.0171 \\
\hline $\mathrm{C} 13$ & $0.0602(2)$ & $0.88460(5)$ & $-0.22590(14)$ & $0.0199(3)$ \\
\hline H13 & -0.0288 & 0.8620 & -0.1797 & $0.024^{*}$ \\
\hline N1 & $0.66548(19)$ & $0.90998(4)$ & $0.10118(11)$ & 0.0152 \\
\hline $\mathrm{N} 2$ & $0.2915(2)$ & $0.97471(5)$ & $-0.40897(12)$ & 0.0214 \\
\hline $\mathrm{H} 2 \mathrm{~A}$ & 0.2825 & 0.9936 & -0.4906 & $0.026^{*}$ \\
\hline $\mathrm{H} 2 \mathrm{~B}$ & 0.1581 & 0.9553 & -0.4100 & $0.026^{*}$ \\
\hline $\mathrm{O} 1$ & $0.71039(17)$ & $1.00283(4)$ & $-0.27249(10)$ & $0.0237(3)$ \\
\hline $\mathrm{O} 2$ & $-0.05271(17)$ & $0.89275(4)$ & $-0.36651(10)$ & 0.0255 \\
\hline
\end{tabular}

Atomic displacement parameters $\left(\AA^{2}\right)$

\begin{tabular}{lllllll}
\hline & $U^{11}$ & $U^{22}$ & $U^{33}$ & $U^{12}$ & $U^{13}$ & $U^{23}$ \\
\hline C1 & $0.0178(6)$ & $0.0173(6)$ & $0.0150(6)$ & $-0.0021(5)$ & $0.0072(5)$ & $0.0043(4)$ \\
C2 & $0.0162(6)$ & $0.0221(7)$ & $0.0233(7)$ & $-0.0031(5)$ & $0.0041(5)$ & $-0.0016(5)$ \\
C3 & $0.0249(7)$ & $0.0193(7)$ & $0.0347(8)$ & $-0.0027(5)$ & $0.0103(6)$ & $-0.0052(5)$
\end{tabular}


supporting information

$\begin{array}{lllllll}\text { C4 } & 0.0234(7) & 0.0199(7) & 0.0380(8) & 0.0052(5) & 0.0120(6) & 0.0061(5) \\ \text { C5 } & 0.0181(6) & 0.0284(7) & 0.0244(7) & 0.0026(5) & 0.0033(5) & 0.0086(5) \\ \text { C6 } & 0.0209(6) & 0.0228(7) & 0.0159(6) & -0.0023(5) & 0.0046(5) & 0.0020(5) \\ \text { C7 } & 0.0167(6) & 0.0198(6) & 0.0113(6) & -0.0003(5) & 0.0029(5) & 0.0011(4) \\ \text { C8 } & 0.0156(6) & 0.0153(6) & 0.0163(6) & -0.0010(4) & 0.0062(5) & 0.0001(4) \\ \text { C9 } & 0.0160(6) & 0.0142(6) & 0.0147(6) & 0.0013(4) & 0.0051(5) & -0.0014(4) \\ \text { C10 } & 0.0153(6) & 0.0175(6) & 0.0154(6) & 0.0012(5) & 0.0057(5) & -0.0008(4) \\ \text { C11 } & 0.0155(6) & 0.0183(6) & 0.0185(6) & -0.0017(5) & 0.0076(5) & 0.0004(5) \\ \text { C12 } & 0.0200(6) & 0.0144(6) & 0.0162(6) & 0.0009(5) & 0.0064(5) & -0.0008(4) \\ \text { C13 } & 0.0171(6) & 0.0221(6) & 0.0194(7) & -0.0005(5) & 0.0059(5) & -0.0007(5) \\ \text { N1 } & 0.0155(5) & 0.0164(5) & 0.0120(5) & 0.0006(4) & 0.0037(4) & 0.0007(4) \\ \text { N2 } & 0.0198(6) & 0.0265(6) & 0.0149(5) & -0.0022(4) & 0.0034(4) & 0.0055(4) \\ \text { O1 } & 0.0233(5) & 0.0275(5) & 0.0177(5) & -0.0062(4) & 0.0052(4) & 0.0042(3) \\ \text { O2 } & 0.0213(5) & 0.0314(6) & 0.0178(5) & -0.0034(4) & 0.0009(4) & -0.0008(4) \\ \end{array}$

Geometric parameters $\left(\AA,{ }^{\circ}\right)$

\begin{tabular}{|c|c|c|c|}
\hline $\mathrm{C} 1-\mathrm{C} 6$ & $1.3942(17)$ & $\mathrm{C} 8-\mathrm{C} 9$ & $1.3709(16)$ \\
\hline $\mathrm{C} 1-\mathrm{C} 2$ & $1.3949(18)$ & $\mathrm{C} 8-\mathrm{N} 1$ & $1.3719(15)$ \\
\hline $\mathrm{C} 1-\mathrm{C} 7$ & $1.5159(16)$ & $\mathrm{C} 8-\mathrm{H} 8$ & 0.9300 \\
\hline $\mathrm{C} 2-\mathrm{C} 3$ & $1.3886(18)$ & $\mathrm{C} 9-\mathrm{C} 10$ & $1.4476(16)$ \\
\hline $\mathrm{C} 2-\mathrm{H} 2$ & 0.9300 & $\mathrm{C} 9-\mathrm{C} 12$ & $1.4834(16)$ \\
\hline $\mathrm{C} 3-\mathrm{C} 4$ & $1.3933(19)$ & $\mathrm{C} 10-\mathrm{C} 11$ & $1.3829(16)$ \\
\hline $\mathrm{C} 3-\mathrm{H} 3$ & 0.9300 & $\mathrm{C} 10-\mathrm{C} 13$ & $1.4471(17)$ \\
\hline $\mathrm{C} 4-\mathrm{C} 5$ & $1.384(2)$ & $\mathrm{C} 11-\mathrm{N} 1$ & $1.3516(15)$ \\
\hline $\mathrm{C} 4-\mathrm{H} 4$ & 0.9300 & $\mathrm{C} 11-\mathrm{H} 11$ & 0.9300 \\
\hline $\mathrm{C} 5-\mathrm{C} 6$ & $1.3877(18)$ & $\mathrm{C} 12-\mathrm{O} 1$ & $1.2375(15)$ \\
\hline $\mathrm{C} 5-\mathrm{H} 5$ & 0.9300 & $\mathrm{C} 12-\mathrm{N} 2$ & $1.3374(16)$ \\
\hline $\mathrm{C} 6-\mathrm{H} 6$ & 0.9300 & $\mathrm{C} 13-\mathrm{O} 2$ & $1.2253(15)$ \\
\hline $\mathrm{C} 7-\mathrm{N} 1$ & $1.4616(14)$ & $\mathrm{C} 13-\mathrm{H} 13$ & 0.9300 \\
\hline C7-H7A & 0.9700 & $\mathrm{~N} 2-\mathrm{H} 2 \mathrm{~A}$ & 0.8600 \\
\hline C7-H7B & 0.9700 & $\mathrm{~N} 2-\mathrm{H} 2 \mathrm{~B}$ & 0.8600 \\
\hline $\mathrm{C} 6-\mathrm{C} 1-\mathrm{C} 2$ & $118.58(11)$ & $\mathrm{C} 9-\mathrm{C} 8-\mathrm{N} 1$ & $109.08(10)$ \\
\hline $\mathrm{C} 6-\mathrm{C} 1-\mathrm{C} 7$ & $119.09(11)$ & $\mathrm{C} 9-\mathrm{C} 8-\mathrm{H} 8$ & 125.5 \\
\hline $\mathrm{C} 2-\mathrm{C} 1-\mathrm{C} 7$ & $122.33(11)$ & $\mathrm{N} 1-\mathrm{C} 8-\mathrm{H} 8$ & 125.5 \\
\hline $\mathrm{C} 3-\mathrm{C} 2-\mathrm{C} 1$ & $120.71(12)$ & $\mathrm{C} 8-\mathrm{C} 9-\mathrm{C} 10$ & $106.34(10)$ \\
\hline $\mathrm{C} 3-\mathrm{C} 2-\mathrm{H} 2$ & 119.6 & $\mathrm{C} 8-\mathrm{C} 9-\mathrm{C} 12$ & $121.46(11)$ \\
\hline $\mathrm{C} 1-\mathrm{C} 2-\mathrm{H} 2$ & 119.6 & $\mathrm{C} 10-\mathrm{C} 9-\mathrm{C} 12$ & $132.19(11)$ \\
\hline $\mathrm{C} 2-\mathrm{C} 3-\mathrm{C} 4$ & $120.19(12)$ & $\mathrm{C} 11-\mathrm{C} 10-\mathrm{C} 13$ & $119.54(11)$ \\
\hline $\mathrm{C} 2-\mathrm{C} 3-\mathrm{H} 3$ & 119.9 & $\mathrm{C} 11-\mathrm{C} 10-\mathrm{C} 9$ & $106.24(10)$ \\
\hline $\mathrm{C} 4-\mathrm{C} 3-\mathrm{H} 3$ & 119.9 & $\mathrm{C} 13-\mathrm{C} 10-\mathrm{C} 9$ & $134.08(11)$ \\
\hline $\mathrm{C} 5-\mathrm{C} 4-\mathrm{C} 3$ & $119.32(12)$ & $\mathrm{N} 1-\mathrm{C} 11-\mathrm{C} 10$ & $109.15(10)$ \\
\hline $\mathrm{C} 5-\mathrm{C} 4-\mathrm{H} 4$ & 120.3 & $\mathrm{~N} 1-\mathrm{C} 11-\mathrm{H} 11$ & 125.4 \\
\hline $\mathrm{C} 3-\mathrm{C} 4-\mathrm{H} 4$ & 120.3 & $\mathrm{C} 10-\mathrm{C} 11-\mathrm{H} 11$ & 125.4 \\
\hline $\mathrm{C} 4-\mathrm{C} 5-\mathrm{C} 6$ & $120.55(12)$ & $\mathrm{O} 1-\mathrm{C} 12-\mathrm{N} 2$ & $122.68(11)$ \\
\hline $\mathrm{C} 4-\mathrm{C} 5-\mathrm{H} 5$ & 119.7 & $\mathrm{O} 1-\mathrm{C} 12-\mathrm{C} 9$ & $120.65(10)$ \\
\hline $\mathrm{C} 6-\mathrm{C} 5-\mathrm{H} 5$ & 119.7 & $\mathrm{~N} 2-\mathrm{C} 12-\mathrm{C} 9$ & $116.67(10)$ \\
\hline
\end{tabular}




$\begin{array}{llll}\mathrm{C} 5-\mathrm{C} 6-\mathrm{C} 1 & 120.65(12) & \mathrm{O} 2-\mathrm{C} 13-\mathrm{C} 10 & 127.90(12) \\ \mathrm{C} 5-\mathrm{C} 6-\mathrm{H} 6 & 119.7 & \mathrm{O} 2-\mathrm{C} 13-\mathrm{H} 13 & 116.0 \\ \mathrm{C} 1-\mathrm{C} 6-\mathrm{H} 6 & 119.7 & \mathrm{C} 10-\mathrm{C} 13-\mathrm{H} 13 & 116.0 \\ \mathrm{~N} 1-\mathrm{C} 7-\mathrm{C} 1 & 112.69(9) & \mathrm{C} 11-\mathrm{N} 1-\mathrm{C} 8 & 109.20(10) \\ \mathrm{N} 1-\mathrm{C} 7-\mathrm{H} 7 \mathrm{~A} & 109.1 & \mathrm{C} 11-\mathrm{N} 1-\mathrm{C} 7 & 126.38(10) \\ \mathrm{C} 1-\mathrm{C} 7-\mathrm{H} 7 \mathrm{~A} & 109.1 & \mathrm{C} 8-\mathrm{N} 1-\mathrm{C} 7 & 124.07(10) \\ \mathrm{N} 1-\mathrm{C} 7-\mathrm{H} 7 \mathrm{~B} & 109.1 & \mathrm{C} 12-\mathrm{N} 2-\mathrm{H} 2 \mathrm{~A} & 120.0 \\ \mathrm{C} 1-\mathrm{C} 7-\mathrm{H} 7 \mathrm{~B} & 109.1 & \mathrm{C} 12-\mathrm{N} 2-\mathrm{H} 2 \mathrm{~B} & 120.0 \\ \mathrm{H} 7 \mathrm{~A}-\mathrm{C} 7-\mathrm{H} 7 \mathrm{~B} & 107.8 & \mathrm{H} 2 \mathrm{~A}-\mathrm{N} 2-\mathrm{H} 2 \mathrm{~B} & 120.0 \\ & & & 6.5(2) \\ \mathrm{C} 6-\mathrm{C} 1-\mathrm{C} 2-\mathrm{C} 3 & -0.61(18) & \mathrm{C} 12-\mathrm{C} 9-\mathrm{C} 10-\mathrm{C} 13 & 176.01(10) \\ \mathrm{C} 7-\mathrm{C} 1-\mathrm{C} 2-\mathrm{C} 3 & 178.65(11) & \mathrm{C} 13-\mathrm{C} 10-\mathrm{C} 11-\mathrm{N} 1 & -0.30(13) \\ \mathrm{C} 1-\mathrm{C} 2-\mathrm{C} 3-\mathrm{C} 4 & 0.9(2) & \mathrm{C} 9-\mathrm{C} 10-\mathrm{C} 11-\mathrm{N} 1 & 3.69(18) \\ \mathrm{C} 2-\mathrm{C} 3-\mathrm{C} 4-\mathrm{C} 5 & -0.3(2) & \mathrm{C} 8-\mathrm{C} 9-\mathrm{C} 12-\mathrm{O} 1 & -178.07(11) \\ \mathrm{C} 3-\mathrm{C} 4-\mathrm{C} 5-\mathrm{C} 6 & -0.6(2) & \mathrm{C} 10-\mathrm{C} 9-\mathrm{C} 12-\mathrm{O} 1 & -176.06(10) \\ \mathrm{C} 4-\mathrm{C} 5-\mathrm{C} 6-\mathrm{C} 1 & 0.92(19) & \mathrm{C} 8-\mathrm{C} 9-\mathrm{C} 12-\mathrm{N} 2 & 2.19(19) \\ \mathrm{C} 2-\mathrm{C} 1-\mathrm{C} 6-\mathrm{C} 5 & -0.30(18) & \mathrm{C} 10-\mathrm{C} 9-\mathrm{C} 12-\mathrm{N} 2 & -173.94(12) \\ \mathrm{C} 7-\mathrm{C} 1-\mathrm{C} 6-\mathrm{C} 5 & -179.59(11) & \mathrm{C} 11-\mathrm{C} 10-\mathrm{C} 13-\mathrm{O} 2 & 1.1(2) \\ \mathrm{C} 6-\mathrm{C} 1-\mathrm{C} 7-\mathrm{N} 1 & 151.87(11) & \mathrm{C} 9-\mathrm{C} 10-\mathrm{C} 13-\mathrm{O} 2 & 0.01(13) \\ \mathrm{C} 2-\mathrm{C} 1-\mathrm{C} 7-\mathrm{N} 1 & -27.39(15) & \mathrm{C} 10-\mathrm{C} 11-\mathrm{N} 1-\mathrm{C} 8 & -173.30(10) \\ \mathrm{N} 1-\mathrm{C} 8-\mathrm{C} 9-\mathrm{C} 10 & -0.47(13) & \mathrm{C} 10-\mathrm{C} 11-\mathrm{N} 1-\mathrm{C} 7 & 0.30(13) \\ \mathrm{N} 1-\mathrm{C} 8-\mathrm{C} 9-\mathrm{C} 12 & 178.17(10) & \mathrm{C} 9-\mathrm{C} 8-\mathrm{N} 1-\mathrm{C} 11 & 173.80(10) \\ \mathrm{C} 8-\mathrm{C} 9-\mathrm{C} 10-\mathrm{C} 11 & 0.47(13) & \mathrm{C} 9-\mathrm{C} 8-\mathrm{N} 1-\mathrm{C} 7 & 90.92(13) \\ \mathrm{C} 12-\mathrm{C} 9-\mathrm{C} 10-\mathrm{C} 11 & -177.97(12) & \mathrm{C} 1-\mathrm{C} 7-\mathrm{N} 1-\mathrm{C} 11 & -81.44(13) \\ \mathrm{C} 8-\mathrm{C} 9-\mathrm{C} 10-\mathrm{C} 13 & -175.05(13) & \mathrm{C} 1-\mathrm{C} 7-\mathrm{N} 1-\mathrm{C} 8 & \\ & & & \end{array}$

Hydrogen-bond geometry $\left(\AA,{ }^{\circ}\right)$

$\mathrm{Cg} 1$ is the centroid of the benzyl ring $\mathrm{C} 1-\mathrm{C} 6$.

\begin{tabular}{lllll}
\hline$D-\mathrm{H} \cdots A$ & $D-\mathrm{H}$ & $\mathrm{H} \cdots A$ & $D \cdots A$ & $D-\mathrm{H} \cdots A$ \\
\hline $\mathrm{N} 2-\mathrm{H} 2 B \cdots \mathrm{O} 2$ & 0.86 & 1.99 & $2.8184(14)$ & 160 \\
$\mathrm{~N} 2-\mathrm{H} 2 A \cdots \mathrm{O} 1^{\mathrm{i}}$ & 0.86 & 2.22 & $3.0063(14)$ & 151 \\
$\mathrm{C} 8-\mathrm{H} 8 \cdots \mathrm{O} 1^{\mathrm{ii}}$ & 0.93 & 2.69 & $3.4252(15)$ & 136 \\
$\mathrm{C} 7-\mathrm{H} 7 B^{\cdots}{ }^{\mathrm{O}} 1^{\mathrm{ii}}$ & 0.97 & 2.48 & $3.3123(15)$ & 144 \\
$\mathrm{C} 7-\mathrm{H} 7 A \cdots \mathrm{O} 2^{\text {iii }}$ & 0.97 & 2.66 & $3.3268(15)$ & 126 \\
$\mathrm{C} 11-\mathrm{H} 11 \cdots C g 1^{\text {iv }}$ & 0.93 & 2.58 & $3.4962(14)$ & 167
\end{tabular}

Symmetry codes: (i) $-x+1,-y+2,-z-1$; (ii) $-x+2,-y+2,-z$; (iii) $x+1, y, z+1$; (iv) $x-1, y, z$. 